\title{
Higher-Order Deflation for Polynomial Systems with Isolated Singular Solutions
}

\author{
Anton Leykin* Jan Verschelde ${ }^{\dagger} \quad$ Ailing Zhao ${ }^{\ddagger}$
}

4 January 2007

\begin{abstract}
Given an approximation to a multiple isolated solution of a polynomial system of equations, we have provided a symbolic-numeric deflation algorithm to restore the quadratic convergence of Newton's method. Using first-order derivatives of the polynomials in the system, our method creates an augmented system of equations which has the multiple isolated solution of the original system as a regular root.

In this paper we consider two approaches to computing the "multiplicity structure" at a singular isolated solution. An idea coming from one of them gives rise to our new higher-order deflation method. Using higherorder partial derivatives of the original polynomials, the new algorithm reduces the multiplicity faster than our first method for systems which require several first-order deflation steps.

We also present an algorithm to predict the order of the deflation. 2000 Mathematics Subject Classification. Primary 65H10. Secondary 14Q99, 68W30.

Key words and phrases. Deflation, isolated singular solutions, Newton's method, multiplicity, polynomial systems, reconditioning, symbolicnumeric computations.
\end{abstract}

\footnotetext{
${ }^{*}$ Department of Mathematics, Statistics, and Computer Science, University of Illinois at Chicago, 851 South Morgan (M/C 249), Chicago, IL 60607-7045, USA. Email: leykin@math.uic.edu. URL: http://www.math.uic.edu/ leykin

${ }^{\dagger}$ Department of Mathematics, Statistics, and Computer Science, University of Illinois at Chicago, 851 South Morgan (M/C 249), Chicago, IL 60607-7045, USA. Email: jan@math.uic.edu or jan.verschelde@na-net.ornl.gov. URL: http://www.math.uic.edu/ jan This material is based upon work supported by the National Science Foundation under Grant No. 0105739 and Grant No. 0134611.

‡Department of Mathematics, Statistics, and Computer Science, University of Illinois at Chicago, 851 South Morgan (M/C 249), Chicago, IL 60607-7045, USA. Email: azhao1@uic.edu. URL: http://www.math.uic.edu/ ${ }^{\sim}$ azhao1
} 


\section{Introduction}

This paper describes a numerical treatment of singular solutions of polynomial systems. A trivial example to consider would be a single equation with a double root, $f(x)=x^{2}=0$, or a cluster of two very close roots, $f(x)=x^{2}-\varepsilon^{2}=0$, where $0<\varepsilon \ll$ machine precision. In both cases getting good approximate solutions with straightforward numerical approaches such as Newton's method is not easy. Instead of attempting to solve the given equations we replace them with the system augmented by the equation's derivative, $\overline{\boldsymbol{f}}(x)=\left(f(x), f^{\prime}(x)\right)=\mathbf{0}$. Note that this completely symbolic procedure leads to a system with exact regular root in the first case, whereas in the second the system $\overline{\boldsymbol{f}}(x)=\mathbf{0}$ would be inconsistent. However, a numerical solver applied to the latter would converge to a regular solution of a close-by system.

In general setting, given an overdetermined system of equations in many variables with a multiple isolated solution (a cluster of solutions) our approach deflates the multiplicity of the solution (cluster) by applying a certain numerical procedure. From the point of view of the numerical analysis it may be called a reconditioning method: to recondition means to reformulate a problem so its condition number improves.

Our deflation method was first presented at 26, and then described in greater detail in [14. In [13, a directed acyclic graph of Jacobian matrices was introduced for an efficient implementation.

On input we consider clusters of approximate zeroes of systems $F(\boldsymbol{x})=$ $\left(f_{1}(\boldsymbol{x}), f_{2}(\boldsymbol{x}), \ldots, f_{N}(\boldsymbol{x})\right)=\mathbf{0}$ of $N$ equations in $n$ unknowns $\boldsymbol{x} \in \mathbb{C}^{n}$. We assume the cluster approximates an isolated solution $\boldsymbol{x}^{*}$ of $F(\boldsymbol{x})=\mathbf{0}$. Therefore, $N \geq n$. As $\boldsymbol{x}^{*}$ is a singular solution, the Jacobian matrix of $F(\boldsymbol{x})$, denoted by $A(\boldsymbol{x})$, is singular at $\boldsymbol{x}^{*}$. In particular, we have $r=\operatorname{Rank}\left(A\left(\boldsymbol{x}^{*}\right)\right)<n$.

In case $r=n-1$, consider a nonzero vector $\boldsymbol{\lambda}$ in the kernel of $A\left(\boldsymbol{x}^{*}\right)$, which we denote by $\boldsymbol{\lambda} \in \operatorname{ker}\left(A\left(\boldsymbol{x}^{*}\right)\right)$, then the equations

$$
g_{i}(\boldsymbol{x})=\sum_{i=1}^{n} \lambda_{j} \frac{\partial f_{i}(\boldsymbol{x})}{\partial x_{j}}, \quad i=1,2, \ldots, N
$$

vanish at $\boldsymbol{x}^{*}$, because $r=\operatorname{Rank}\left(A\left(\boldsymbol{x}^{*}\right)\right)<n$. For $r<n-1$, our algorithm reduces to the corank-1 case, replacing $A(\boldsymbol{x})$ by $A(\boldsymbol{x}) B$, where $B$ is a random complex $N$-by- $(r+1)$ matrix. For the uniqueness $\boldsymbol{\lambda} \in \operatorname{ker}\left(A\left(\boldsymbol{x}^{*}\right)\right)$, we add a linear scaling equation $\langle\boldsymbol{h}, \boldsymbol{\lambda}\rangle=1$ (using a random complex $(r+1)$-vector $\boldsymbol{h}$ ). and consider the augmented system

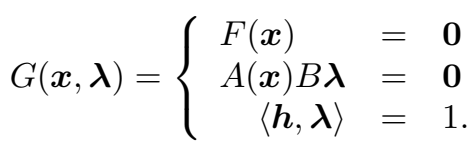

Let us denote by $\mu_{F}\left(\boldsymbol{x}^{*}\right)$ the multiplicity of $\boldsymbol{x}^{*}$ as a solution of the system $F(\boldsymbol{x})=0$. In [14] we proved that there is a $\boldsymbol{\lambda}^{*}$ such that $\mu_{G}\left(\boldsymbol{x}^{*}, \boldsymbol{\lambda}^{*}\right)<\mu_{F}\left(\boldsymbol{x}^{*}\right)$. 
Therefore, our deflation algorithm takes at most $m-1$ stages to determine $\boldsymbol{x}^{*}$ as a regular root of an augmented polynomial system.

Related work. The literature on Newton's method is vast. As stated in [5], Lyapunov-Schmidt reduction (see also [6, §6.2], 11, 11, and [15)) stands at the beginning of every mathematical treatment of singularities. We found the inspiration to develop a symbolic-numeric deflation algorithm in 19 . The symbolic deflation procedure of [12] restores the quadratic convergence of Newton's method with a complexity proportional to the square of the multiplicity of the root. Algorithms to compute the multiplicity are presented in [2], 3], and [23].

Our Contributions. We establish the link between two different objects describing what we call the multiplicity structure of an isolated singular solution: the dual space of differential functionals and the initial ideal with respect to a local monomial order, both associated to the ideal generated by the polynomials in the system in the polynomial ring.

Next, following the latter method, we explain how to compute a basis of the dual space, first, following the ideas of Dayton and Zeng [3], then using the approach of Stetter and Thallinger 23. We provide a formal symbolic algorithm for each approach, respectively called the DZ and ST algorithms; the ingredients of the algorithms do not go beyond linear algebra. Moreover, we present an algorithm to determine the order of the deflation.

The formalism developed for DZ and ST algorithms found a natural continuation in higher-order deflation method that generalizes and extends the firstorder deflation in 14. For the systems that require more than one deflation step by our first algorithm, the new deflation algorithm is capable of completing the deflation in fewer steps.

Acknowledgements. The material in this paper was presented by the first two authors at the workshops on computational algebraic geometry and realnumber complexity, organized respectively by Teresa Krick \& Andrei Gabrielov and Peter Buergisser \& Gregorio Malajovich. The authors thank the organizers for the opportunities to present their work at these FoCM 2005 workshops.

\section{Statement of the Main Theorem \& Algorithms}

The matrices $A^{(d)}(\boldsymbol{x})$ we introduce below coincide for $d=1$ with the Jacobian matrix of a polynomial system. They are generalizations of the Jacobian matrix, built along the same construction as the matrices used in the computation of the multiplicity by Dayton and Zeng in $\underline{3}$.

Definition 2.1 The deflation matrix $A^{(d)}(\boldsymbol{x})$ of a polynomial system $F=\left(f_{1}\right.$, $\left.f_{2}, \ldots, f_{N}\right)$ of $N$ equations in $n$ unknowns $\boldsymbol{x}=\left(x_{1}, x_{2}, \ldots, x_{n}\right)$ is a matrix with elements in $\mathbb{C}[\boldsymbol{x}]$. The rows of $A^{(d)}(\boldsymbol{x})$ are indexed by $\boldsymbol{x}^{\alpha} f_{j}$, where $|\alpha|<d$ and $j=1,2, \ldots, N$. The columns are indexed by partial differential operators $\boldsymbol{\partial}^{\beta}=\frac{\partial^{|\beta|}}{\partial x_{1}^{\beta 1} \ldots \partial x_{n}^{\beta n}}$, where $\beta \neq \mathbf{0}$ and $|\beta| \leq d$. The element at row $\boldsymbol{x}^{\alpha} f_{j}$ and 
column $\partial^{\beta}$ of $A^{(d)}(\boldsymbol{x})$ is

$$
\partial^{\beta} \cdot\left(\boldsymbol{x}^{\alpha} f_{j}\right)=\frac{\partial^{|\beta|}\left(\boldsymbol{x}^{\alpha} f_{j}\right)}{\partial \boldsymbol{x}^{\beta}} .
$$

$A^{(d)}(\boldsymbol{x})$ has $N_{r}$ rows and $N_{c}$ columns, $N_{r}=N \cdot\left(\begin{array}{c}n+d-1 \\ n\end{array}\right)$ and $N_{c}=\left(\begin{array}{c}n+d \\ n\end{array}\right)-1$.

Example 2.2 (Second-order deflation matrix) Consider a system of 3 equations in 2 variables $F=\left(f_{1}, f_{2}, f_{3}\right)=\mathbf{0}$, where $f_{1}=x_{1}^{2}, f_{2}=x_{1}^{2}-x_{2}^{3}$, and $f_{3}=x_{2}^{4}$. Then the second-order deflation matrix $A^{(2)}(\boldsymbol{x})$ of $f$ is

\begin{tabular}{|c|c|c|c|c|c|}
\hline & $\partial_{x_{1}}$ & $\partial_{x_{2}}$ & $\partial_{x_{1}}^{2}$ & $\partial_{x_{1}} \partial_{x_{2}}$ & $\partial_{x_{2}}^{2}$ \\
\hline$f_{1}$ & $2 x_{1}$ & 0 & 2 & 0 & 0 \\
\hline$f_{2}$ & $2 x_{1}$ & $-3 x_{2}^{2}$ & 2 & 0 & $-6 x_{2}$ \\
\hline$f_{3}$ & 0 & $4 x_{2}^{3}$ & 0 & 0 & $12 x_{2}^{2}$ \\
\hline$x_{1} f_{1}$ & $3 x_{1}^{2}$ & 0 & $6 x_{1}$ & 0 & 0 \\
\hline$x_{1} f_{2}$ & $3 x_{1}^{2}$ & $-3 x_{1} x_{2}^{2}$ & $6 x_{1}$ & $3 x_{2}^{2}$ & $-6 x_{1} x_{2}$ \\
\hline$x_{1} f_{3}$ & $x_{2}^{4}$ & $4 x_{1} x_{2}^{3}$ & 0 & 0 & 0 \\
\hline$x_{2} f_{1}$ & $2 x_{1} x_{2}$ & $x_{1}^{2}$ & $2 x_{2}$ & $2 x_{1}$ & 0 \\
\hline$x_{2} f_{2}$ & $2 x_{1} x_{2}$ & $-4 x_{2}^{3}$ & $2 x_{2}$ & $2 x_{1}$ & $-12 x_{2}^{2}$ \\
\hline$x_{2} f_{3}$ & 0 & $5 x_{2}^{4}$ & 0 & 0 & $20 x_{2}^{3}$ \\
\hline
\end{tabular}

Notice that $A^{(1)}(\boldsymbol{x})$ (or the Jacobian matrix of $F$ ) is contained in the first three rows and two columns of $A^{(2)}(\boldsymbol{x})$.

Definition 2.3 Let $\boldsymbol{x}^{*}$ be an isolated singular solution of the system $F(\boldsymbol{x})=\mathbf{0}$ and let $d$ be the order of the deflation. Take a nonzero $N_{r}$-vector $\left(\lambda_{\beta}\right)_{\beta \neq \mathbf{0},|\beta| \leq d}$ in the kernel of $A^{(d)}\left(\boldsymbol{x}^{*}\right)$. It corresponds to what we call a deflation operator a linear differential operator with constant coefficients $\lambda_{\beta}$

$$
Q=\sum_{\beta \neq \mathbf{0},|\beta| \leq d} \lambda_{\beta} \partial^{\beta} \in \mathbb{C}[\boldsymbol{\partial}] .
$$

We use $Q$ to define $N_{r}$ new equations

$$
g_{j, \alpha}(\boldsymbol{x})=Q \cdot\left(\boldsymbol{x}^{\alpha} f_{j}\right)=0, \quad j=1,2, \ldots, N,|\alpha|<d .
$$

When we consider $\lambda_{\beta}$ as indeterminate, we write $g_{j, \alpha}$ as $g_{j, \alpha}(\boldsymbol{x}, \boldsymbol{\lambda})$. In that case, we define $m$ additional linear equations, for $m=\operatorname{corank}\left(A^{(d)}\left(\boldsymbol{x}^{*}\right)\right)$ :

$$
h_{k}(\boldsymbol{\lambda})=\sum_{\beta} b_{k, \beta} \lambda_{\beta}-1=0, \quad k=1,2, \ldots, m,
$$

where the coefficients $b_{k, \beta}$ are randomly chosen complex numbers.

Now we are ready to state our main theorem. 
Theorem 2.4 Let $\boldsymbol{x}^{*} \in \mathbb{C}^{n}$ be an isolated solution of $F(\boldsymbol{x})=\mathbf{0}$. Consider the following system in $\mathbb{C}[\boldsymbol{x}, \boldsymbol{\lambda}]$ :

$$
G^{(d)}(\boldsymbol{x}, \boldsymbol{\lambda})=\left\{\begin{aligned}
f_{j}(\boldsymbol{x}) & =0, \quad j=1,2, \ldots, N \\
g_{j, \alpha}(\boldsymbol{x}, \boldsymbol{\lambda}) & =0, \quad j=1,2, \ldots, N,|\alpha|<d \\
h_{k}(\boldsymbol{\lambda}) & =0, \quad k=1,2, \ldots, m
\end{aligned}\right.
$$

For a generic choice of coefficients $b_{k, \beta}$, there exists a unique $\boldsymbol{\lambda}^{*} \in \mathbb{C}^{N_{c}}$ such that the system $G^{(d)}(\boldsymbol{x}, \boldsymbol{\lambda})$ has an isolated solution at $\left(\boldsymbol{x}^{*}, \boldsymbol{\lambda}^{*}\right)$. Moreover, the multiplicity of $\left(\boldsymbol{x}^{*}, \boldsymbol{\lambda}^{*}\right)$ in $G(\boldsymbol{x}, \boldsymbol{\lambda})=\mathbf{0}$ is strictly less than the multiplicity of $\boldsymbol{x}^{*}$ in $F(\boldsymbol{x})=\mathbf{0}$.

To determine the order $d$, we propose Algorithm 2.5. This $d$ is then used in Algorithm 2.6.

Algorithm 2.5 d $=\operatorname{MinOrderForCorankDrop}\left(F, x_{0}\right)$

Input: $\quad F$ is a finite set of polynomials;

$\boldsymbol{x}^{0} \approx \boldsymbol{x}^{*}, \boldsymbol{x}^{*}$ is an isolated multiple solution of $F(\boldsymbol{x})=\mathbf{0}$.

Output: $\quad d$ is the minimal number such that the augmented system $G^{(d)}$

produced via a generic deflation operator $Q$ of order $d$ has corank of the Jacobian at $\boldsymbol{x}^{*}$ lower than corank $A\left(\boldsymbol{x}^{*}\right)$.

take a generic vector $\gamma=\left(\gamma_{1}, \ldots, \gamma_{n}\right) \in \operatorname{ker} A\left(\boldsymbol{x}^{0}\right)$;

let $H(t)=F\left(\boldsymbol{x}^{0}+\gamma t\right)=F\left(x_{1}^{0}+\gamma_{1} t, \ldots, x_{n}^{0}+\gamma_{n} t\right)$;

$d:=\min \{a \mid a$ belongs to the support of $H(t)\}-1$.

Algorithm 2.6 $D^{(d)} F=\operatorname{Deflate}\left(F, d, \boldsymbol{x}_{0}\right)$

Input: $\quad F$ is a finite set of polynomials in $\mathbb{C}[\boldsymbol{x}]$;

$d$ is the order of deflation;

$\boldsymbol{x}^{0} \approx \boldsymbol{x}^{*}, \boldsymbol{x}^{*}$ is an isolated multiple solution of $F(\boldsymbol{x})=\mathbf{0}$.

Output: $\quad D^{(d)} F$ is a finite set of polynomials in $\mathbb{C}[\boldsymbol{x}, \boldsymbol{\lambda}]$

such that there is $\boldsymbol{\lambda}^{*}$ with $\mu_{D^{(d)} F}\left(\boldsymbol{x}^{*}, \boldsymbol{\lambda}^{*}\right)<\mu_{F}\left(\boldsymbol{x}^{*}\right)$.

determine the numerical corank $m$ of $A^{(d)}\left(\boldsymbol{x}^{0}\right)$;

return $D^{(d)} F:=G^{(d)}(\boldsymbol{x}, \boldsymbol{\lambda})$ as in (8).

\section{Multiplicity Structure}

This section relates two different ways to obtain the multiplicity of an isolated solution, constructing its multiplicity structure. Note that by a "multiplicity structure" - a term without a precise mathematical definition - we mean any structure which provides more local information about the singular solution in addition to its multiplicity. In this section we mention two different approaches to describe this so-called multiplicity structure. 
Example 3.1 (Running example 1) Consider the system

$$
F(\boldsymbol{x})=\left\{\begin{array}{c}
x_{2}^{3}=0 \\
x_{1}^{2} x_{2}^{2}=0 \\
x_{1}^{4}+x_{1}^{3} x_{2}=0 .
\end{array}\right.
$$

The system $F(\boldsymbol{x})=\mathbf{0}$ has only one isolated solution at $(0,0)$ of high multiplicity. Below we will show how to compute the multiplicity of $(0,0)$.

\subsection{Standard Bases}

Assume $\mathbf{0} \in \mathbb{C}^{n}$ is an isolated solution of the system $F(\boldsymbol{x})=\mathbf{0}$. Let $I=\langle F\rangle \subset$ $R=\mathbb{C}[\boldsymbol{x}]$ be the ideal generated by the polynomials in the system. Given a local monomial order $\geq$, the initial ideal $\operatorname{in}_{\geq}(I)=\left\{\operatorname{in}_{\geq}(f) \mid f \in I\right\} \subset R$ describes the multiplicity structure of $\mathbf{0}$ by means of standard monomials, i.e.: monomials that are not contained in $\operatorname{in}_{\geq}(I)$. A graphical representation of a monomial ideal is a monomial staircase.

Example 3.2 (Initial ideals with respect to a local order) Consider the system (9) of Example 3.1.

Figure 1 shows the staircases for initial ideals of $I=\langle F\rangle$ w.r.t. two local weight orders $\geq_{\omega}$. Computer algebra systems Macaulay 2 [7] and Singular [10] can be used for these kind of computations, see also [8, 9] for theory, in particular, on Mora's tangent cone algorithm [17.

In the example the leading monomials at the corners of the staircase come from the elements of the corresponding standard basis. For the weight vector $w=(-1,-2)$ the original generators give such a basis (initial terms underlined). For $w=(-2,-1)$ one more polynomial is needed.
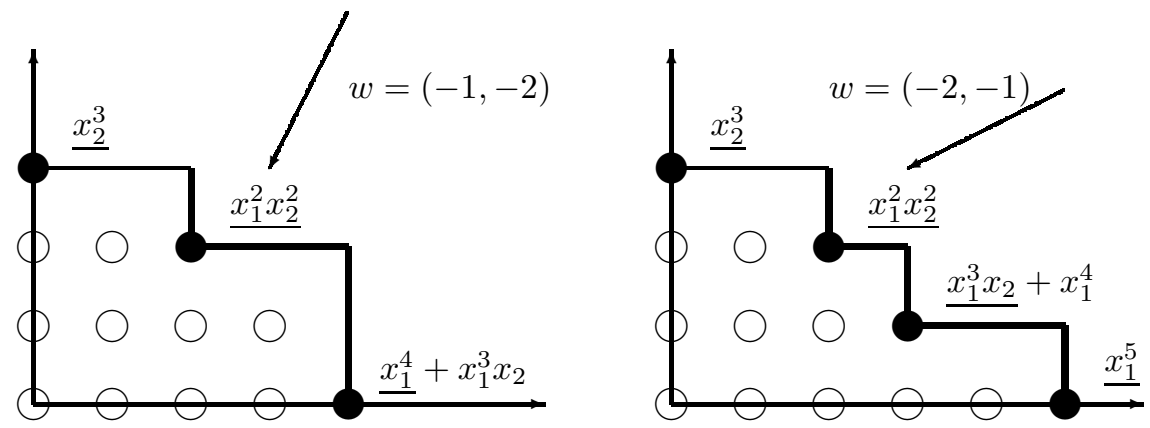

Figure 1: Two monomial staircases for two different monomial orderings applied to the same system. The full circles represent the generators of the initial ideals. The multiplicity is the number of standard monomials, represented by the empty circles under the staircase. 


\subsection{Dual Space of Differential Functionals}

Another approach at the multiplicity structure is described in detail in 22, 24]; see also [18. Using duality to define the multiplicity of a solution goes back to Macaulay [16. In this approach, differential functionals are denoted by

$$
\Delta_{\alpha}(f)=\left.\frac{1}{\alpha_{1} ! \cdots \alpha_{n} !} \cdot \frac{\partial^{|\alpha|} f}{\partial x^{\alpha_{1}} \cdots \partial x^{\alpha_{n}}}\right|_{\boldsymbol{x}=\mathbf{0}} .
$$

Observe that

$$
\Delta_{\alpha}\left(\boldsymbol{x}^{\beta}\right)= \begin{cases}1, & \alpha=\beta \\ 0, & \alpha \neq \beta\end{cases}
$$

We then define the local dual space of differential functionals $D_{\mathbf{0}}[I]$ as

$$
D_{0}[I]=\left\{L \in \operatorname{Span}\left\{\Delta_{\alpha} \mid \alpha \in \mathbb{Z}_{\geq 0}^{n}\right\} \mid L(f)=0 \text { for all } f \in I\right\},
$$

Example 3.3 (Dual space of running example 1) For the ideal defined by the polynomials in the system (9) we have

$$
\begin{aligned}
& D_{0}[I]=\operatorname{Span}\left\{\quad \underline{\Delta_{(4,0)}}-\Delta_{(3,1)}, \underline{\Delta_{(3,0)}}, \underline{\Delta_{(2,1)}}, \underline{\Delta_{(1,2)}},\right. \\
& \left.\underline{\Delta_{(2,0)}}, \underline{\Delta_{(1,1)}}, \underline{\Delta_{(0,2)}}, \underline{\Delta_{(1,0)}}, \underline{\Delta_{(0,1)}}, \underline{\Delta_{(0,0)}}\right\} .
\end{aligned}
$$

Notice that here the basis of the dual space is chosen in such a way that the (underlined) leading terms with respect to the weight order $\geq_{(2,1)}$ correspond to the monomials under the staircase in Example 3.1 for the order $\geq_{(-2,-1)}$. We will show that it is not a coincidence later in this section.

\subsection{Dual Bases versus Standard Bases}

Since both local dual bases and initial ideals w.r.t. local orders describe the same, there exists a natural correspondence between the two.

Let $\geq$ be an order on the nonnegative integer lattice $\mathbb{Z}_{\geq 0}^{n}$ that defines a local monomial order and let $\succeq$ be the opposite of $\geq$ : i.e. $\alpha \succeq \bar{\beta} \Leftrightarrow \alpha \leq \beta$. (Note: $\succeq$ defines a global monomial order.)

For a linear differential functional $L=\sum c_{\alpha} \Delta_{\alpha}$ define the support: $\operatorname{supp}(L)=$ $\left\{\alpha \in \mathbb{Z}_{\geq 0}^{n} \mid c_{\alpha} \neq 0\right\}$. For the dual space, $\operatorname{supp}\left(D_{\mathbf{0}}[I]\right)=\bigcup_{L \in D_{\mathbf{0}}[I]} \operatorname{supp}(L)$.

Using the order $\succeq$ we can talk about the leading or initial term of $L$ : let $\operatorname{in}_{\succeq}(L)$ be the maximal element of $\operatorname{supp}(L)$ with respect to $\succeq$. Define the initial support of the dual space as $\operatorname{in}_{\succeq}\left(D_{0}[I]\right)=\left\{\operatorname{in}_{\succeq}(L) \mid L \in D_{0}[I]\right\}$. The initial support is obviously contained in the support, in our running example the containment is proper:

$$
\begin{aligned}
\operatorname{in}_{(2,1)}\left(D_{\mathbf{0}}[I]\right) & =\{(i, j) \mid i+j \leq 3\} \cup\{(4,0)\} \\
& \subset\{(i, j) \mid i+j \leq 3\} \cup\{(4,0) \cup(3,1)\}=\operatorname{supp}\left(D_{\mathbf{0}}[I]\right) .
\end{aligned}
$$


Theorem 3.4 The number of elements in the initial support equals the dimension of the dual space, therefore, is the multiplicity. Moreover, with the above assumptions on the orders $\geq$ and $\succeq$, the standard monomials w.r.t. the local order $\geq$ are $\left\{\boldsymbol{x}^{\alpha} \mid \alpha \in \operatorname{in}_{\succeq}\left(D_{\mathbf{0}}[I]\right)\right\}$.

Proof. Pick $L_{\beta} \in D_{\mathbf{0}}[I], \beta \in \operatorname{in}_{\succeq}\left(D_{\mathbf{0}}[I]\right)$ such that $\operatorname{in}_{\succeq}\left(L_{\beta}\right)=\beta$. One can easily show that $\left\{L_{\beta}\right\}$ is a basis of $D_{0}[I]$.

Take a monomial $\boldsymbol{x}^{\alpha} \in \operatorname{in}_{\geq}(I)$, then there is $f \in I$ such that $\boldsymbol{x}^{\alpha}=\operatorname{in}_{\geq}(f)$. Next, take any linear differential functional $L$ with $\operatorname{in}_{\succeq}(L)=\alpha$. Since the orders $\geq$ and $\succeq$ are opposite, there are no similar terms in the tail of $L$ and the tail of $f$, therefore, $L(f)=\operatorname{in}_{\succeq}(L)\left(\operatorname{in}_{\geq}(f)\right) \neq 0$.

It follows that, $L \notin D_{0}[I]$, which proves that the set of standard monomials is contained in the initial support of $D_{\mathbf{0}}[I]$. They are equal since they both determine the dimension.

Consider the ring of linear differential operators $\mathcal{D}=\mathbb{C}[\boldsymbol{\partial}]$ with the natural action (denoted by ".") on polynomial ring $R=\mathbb{C}[\boldsymbol{x}]$.

Lemma 3.5 Let $Q \in \mathbb{C}[\boldsymbol{\partial}]$ and $f \in \mathbb{C}[\boldsymbol{x}]$ such that $\operatorname{in}_{\succeq}(Q) \succeq \operatorname{in}_{\geq}(f)$ (in $\mathbb{Z}_{\geq 0}^{n}$ ).

Then $\operatorname{in}_{\geq}(Q \cdot f)=\operatorname{in}_{\geq}(f)-\operatorname{in}_{\succeq}(Q) \in \mathbb{Z}_{\geq 0}^{n}$.

\section{Computing the Multiplicity Structure}

Let the ideal $I$ be generated by $f_{1}, f_{2}, \ldots, f_{N}$. Let $D_{\mathbf{0}}^{(d)}[I]$ the part of $D_{\mathbf{0}}[I]$ containing functionals of order at most $d$. We would like to have a criterion that for the differential functional $L$ of degree at most $d$ guarantees $L \in D_{\mathbf{0}}^{(d)}[I]$.

Below we describe two such criteria referred to as closedness conditions; their names are arranged to match the corresponding computational techniques of Dayton-Zeng [3] and Stetter-Thallinger [23] that we will describe later respectively as the DZ and ST algorithms.

A functional $L=\sum c_{\alpha} \Delta_{\alpha}$ with $c_{\alpha} \in \mathbb{C}$ of order $d$ belongs to the dual space $D_{\mathbf{0}}[I]$ if and only if

- (DZ-closedness) $L\left(g \cdot f_{i}\right)=0$ for all $i=1,2, \ldots, N$ and polynomials $g(\boldsymbol{x})$ of degree at most $d-1$.

- (ST-closedness) $L\left(f_{i}\right)=0$ for all $i$ and $\sigma_{j}(L) \in D_{\mathbf{0}}[I]$ for all $j=$ $1,2, \ldots, n$, where $\sigma_{j}: D_{\mathbf{0}}[I] \rightarrow D_{\mathbf{0}}[I]$ is a linear map such that

$$
\sigma_{j}\left(\Delta_{\alpha}\right)=\left\{\begin{array}{cc}
0, & \text { if } \alpha_{j}=0 \\
\Delta_{\alpha-e_{j}}, & \text { otherwise. }
\end{array}\right.
$$

The basic idea of both DZ and ST algorithms is the same: build up a basis of $D_{\mathbf{0}}$ incrementally by computing $D_{\mathbf{0}}^{(d)}$ for $d=1,2, \ldots$ using the corresponding closedness condition. The computation stops when $D_{\mathbf{0}}^{(d)}=D_{\mathbf{0}}^{(d-1)}$. 
Example 4.1 (Running example 2) Consider the system in $\mathbb{C}\left[x_{1}, x_{2}\right]$ given by three polynomials $f_{1}=x_{1} x_{2}, f_{2}=x_{1}^{2}-x_{2}^{2}$, and $f_{3}=x_{2}^{4}$, which has only one isolated root at $(0,0)$.

\subsection{The Dayton-Zeng Algorithm}

We shall outline only a summary of this approach, see [3] for details.

If $\mathbf{0}$ is a solution of the system, then $D_{\mathbf{0}}^{(0)}=\operatorname{Span}\left\{\Delta_{\mathbf{0}}\right\}$.

At step $d>0$, we compute $D_{\mathbf{0}}^{(d)}$. Let the functional

$$
L=\sum_{|\alpha| \leq d, \alpha \neq \mathbf{0}} c_{\alpha} \Delta_{\alpha}
$$

belong to the dual space $D_{0}^{(d)}$. Then the vector of coefficients $c_{\alpha}$ is in the kernel of the following matrix $M_{D Z}^{(d)}$ with $N B(d-1)$ rows and $B(d)-1$ columns, where $B(d)=\left(\begin{array}{c}n+d \\ n\end{array}\right)$ is the number of monomials in $n$ variables of degree at most $d$.

The rows of $M_{D Z}^{(d)}$ are labelled with $x^{\alpha} f_{j}$, where $|\alpha|<d$ and $j=1,2, \ldots, N$. The columns correspond to $\Delta_{\beta}$, where $\beta \neq \mathbf{0},|\beta| \leq d$.

[The entry of $M_{D Z}^{(d)}$ in row $x^{\alpha} f_{j}$ and column $\left.\Delta_{\beta}\right]=\Delta_{\beta}\left(x^{\alpha} f_{j}\right)$.

At the step $d=3$ we have the following $M_{D Z}^{(3)}$

\begin{tabular}{c||cc|ccc|cccc} 
& $\Delta_{(1,0)}$ & $\Delta_{(0,1)}$ & $\Delta_{(2,0)}$ & $\Delta_{(1,1)}$ & $\Delta_{(0,2)}$ & $\Delta_{(3,0)}$ & $\Delta_{(2,1)}$ & $\Delta_{(1,2)}$ & $\Delta_{(0,3)}$ \\
\hline \hline$f_{1}$ & 0 & 0 & 0 & 1 & 0 & 0 & 0 & 0 & 0 \\
$f_{2}$ & 0 & 0 & 1 & 0 & -1 & 0 & 0 & 0 & 0 \\
$f_{3}$ & 0 & 0 & 0 & 0 & 0 & 0 & 0 & 0 & 0 \\
\hline$x_{1} f_{1}$ & 0 & 0 & 0 & 0 & 0 & 0 & 1 & 0 & 0 \\
$x_{1} f_{2}$ & 0 & 0 & 0 & 0 & 0 & 1 & 0 & -1 & 0 \\
$x_{1} f_{3}$ & 0 & 0 & 0 & 0 & 0 & 0 & 0 & 0 & 0 \\
$x_{2} f_{1}$ & 0 & 0 & 0 & 0 & 0 & 0 & 0 & 1 & 0 \\
$x_{2} f_{2}$ & 0 & 0 & 0 & 0 & 0 & 0 & 1 & 0 & -1 \\
$x_{2} f_{3}$ & 0 & 0 & 0 & 0 & 0 & 0 & 0 & 0 & 0 \\
\hline$x_{1}^{2} f_{1}$ & 0 & 0 & 0 & 0 & 0 & 0 & 0 & 0 & 0 \\
$\vdots$ & $\vdots$ & $\vdots$ & $\vdots$ & $\vdots$ & $\vdots$ & $\vdots$ & $\vdots$ & $\vdots$ & $\vdots$
\end{tabular}

Note that the last block of 9 rows is entirely zero.

Analyzing the kernel of this matrix one sees that there are no functionals of degree 3 in the dual space, which is then is equal to $D_{\mathbf{0}}^{(2)}[I]$

$$
D_{0}[I]=\operatorname{Span}\left\{\Delta_{(0,0)}, \Delta_{(1,0)}, \Delta_{(0,1)}, \Delta_{(2,0)}+\Delta_{(0,2)}\right\} .
$$

\subsection{The Stetter-Thallinger Algorithm}

The matrix $M_{S T}^{(d)}$ is a matrix consisting of $n+1$ blocks stacked on top of each other: 
- The top block contains the first $N$ rows of $M_{D Z}^{(d)}$;

- For every $j=1,2, \ldots, n$, let $S_{j}^{(d)}$ be the $(B(d-1)-1) \times(B(d)-1)$-matrix for the linear map

$$
\sigma_{j}: D_{\mathbf{0}}^{(d)} / \operatorname{Span}\left\{\Delta_{\mathbf{0}}\right\} \rightarrow D_{\mathbf{0}}^{(d-1)} / \operatorname{Span}\left\{\Delta_{\mathbf{0}}\right\}
$$

w.r.t. standard bases of functionals.

The block $M_{S T}^{(d-1)} S_{j}$ represents the closedness condition for the "antiderivation" $\sigma_{j}$.

Let us go through the steps of the algorithm for the Example 4.1 .

Step 1. At the beginning we have $M_{S T}^{(1)}$ equal to

\begin{tabular}{c||cc} 
& $\Delta_{(1,0)}$ & $\Delta_{(0,1)}$ \\
\hline \hline$f_{1}$ & 0 & 0 \\
$f_{2}$ & 0 & 0 \\
$f_{3}$ & 0 & 0
\end{tabular}

Therefore, $D_{\mathbf{0}}^{(1)}=\operatorname{Span}\left\{\Delta_{(0,0)}, \Delta_{(1,0)}, \Delta_{(0,1)}\right\}$.

Step 2. Since $M_{S T}^{(1)} S_{j}^{(2)}=0$ for all $j$, the matrix $M_{S T}^{(2)}$ is

\begin{tabular}{c||cc|ccc} 
& $\Delta_{(1,0)}$ & $\Delta_{(0,1)}$ & $\Delta_{(2,0)}$ & $\Delta_{(1,1)}$ & $\Delta_{(0,2)}$ \\
\hline \hline$f_{1}$ & 0 & 0 & 0 & 1 & 0 \\
$f_{2}$ & 0 & 0 & 1 & 0 & -1 \\
$f_{3}$ & 0 & 0 & 0 & 0 & 0 \\
\hline & 0 & 0 & 0 & 0 & 0 \\
$\vdots$ & $\vdots$ & $\vdots$ & $\vdots$ & $\vdots$ & $\vdots$
\end{tabular}

Therefore, $D_{\mathbf{0}}^{(2)}=\operatorname{Span}\left\{\Delta_{(0,0)}, \Delta_{(1,0)}, \Delta_{(0,1)}, \Delta_{(2,0)}+\Delta_{(0,2)}\right\}$.

We can "prune" the matrix $M_{S T}^{(2)}$ by row-reducing it to the following matrix with the same kernel:

$$
\tilde{M}_{S T}^{(2)}=\left[\begin{array}{ccccc}
0 & 0 & 0 & 1 & 0 \\
0 & 0 & 1 & 0 & -1
\end{array}\right]
$$

Step 3. Compute $S_{1}^{(3)}$ that represents $\sigma_{1}$ :

\begin{tabular}{c||cc|ccc|cccc} 
& $\Delta_{(1,0)}$ & $\Delta_{(0,1)}$ & $\Delta_{(2,0)}$ & $\Delta_{(1,1)}$ & $\Delta_{(0,2)}$ & $\Delta_{(3,0)}$ & $\Delta_{(2,1)}$ & $\Delta_{(1,2)}$ & $\Delta_{(0,3)}$ \\
\hline \hline$\Delta_{(1,0)}$ & 0 & 0 & 1 & 0 & 0 & 0 & 0 & 0 & 0 \\
$\Delta_{(0,1)}$ & 0 & 0 & 0 & 1 & 0 & 0 & 0 & 0 & 0 \\
\hline$\Delta_{(2,0)}$ & 0 & 0 & 0 & 0 & 0 & 1 & 0 & 0 & 0 \\
$\Delta_{(1,1)}$ & 0 & 0 & 0 & 0 & 0 & 0 & 1 & 0 & 0 \\
$\Delta_{(0,2)}$ & 0 & 0 & 0 & 0 & 0 & 0 & 0 & 1 & 0
\end{tabular}


The matrix $S_{2}^{(3)}$ can be defined similarly.

The top block of the matrix $M_{S T}^{(3)}$ is

\begin{tabular}{c||cc|ccc|cccc} 
& $\Delta_{(1,0)}$ & $\Delta_{(0,1)}$ & $\Delta_{(2,0)}$ & $\Delta_{(1,1)}$ & $\Delta_{(0,2)}$ & $\Delta_{(3,0)}$ & $\Delta_{(2,1)}$ & $\Delta_{(1,2)}$ & $\Delta_{(0,3)}$ \\
\hline \hline$f_{1}$ & 0 & 0 & 0 & 1 & 0 & 0 & 0 & 0 & 0 \\
$f_{2}$ & 0 & 0 & 1 & 0 & -1 & 0 & 0 & 0 & 0 \\
$f_{3}$ & 0 & 0 & 0 & 0 & 0 & 0 & 0 & 0 & 0
\end{tabular}

Despite the last 4 columns being 0 , there are no new elements of order 3 in the dual space due to the other two blocks: $\tilde{M}_{S T}^{(2)} S_{1}^{(3)}$ :

\begin{tabular}{c||cc|ccc|cccc} 
& $\Delta_{(1,0)}$ & $\Delta_{(0,1)}$ & $\Delta_{(2,0)}$ & $\Delta_{(1,1)}$ & $\Delta_{(0,2)}$ & $\Delta_{(3,0)}$ & $\Delta_{(2,1)}$ & $\Delta_{(1,2)}$ & $\Delta_{(0,3)}$ \\
\hline \hline$x_{1} f_{1}$ & 0 & 0 & 0 & 0 & 0 & 0 & 1 & 0 & 0 \\
$x_{1} f_{2}$ & 0 & 0 & 0 & 0 & 0 & 1 & 0 & -1 & 0
\end{tabular}

and $\tilde{M}_{S T}^{(2)} S_{2}^{(3)}$ :

\begin{tabular}{c||cc|ccc|cccc} 
& $\Delta_{(1,0)}$ & $\Delta_{(0,1)}$ & $\Delta_{(2,0)}$ & $\Delta_{(1,1)}$ & $\Delta_{(0,2)}$ & $\Delta_{(3,0)}$ & $\Delta_{(2,1)}$ & $\Delta_{(1,2)}$ & $\Delta_{(0,3)}$ \\
\hline \hline$x_{2} f_{1}$ & 0 & 0 & 0 & 0 & 0 & 0 & 0 & 1 & 0 \\
$x_{2} f_{2}$ & 0 & 0 & 0 & 0 & 0 & 0 & 1 & 0 & -1
\end{tabular}

Comparing to DZ algorithm, in step 3, we managed to avoid the computation of 9 last zero rows of $M_{D Z}^{(3)}$ in this particular example. We now also see how its 4 last nonzero rows show up in the "closedness condition" blocks of $M_{D Z}^{(3)}$.

\section{Proofs and Algorithmic Details}

In this section we justify the main theorems stated before and give details about the algorithms presented above.

\subsection{First-Order Deflation}

In this section we summarize our deflation method introduced in [14. Not only it is done for the convenience of the reader, but also for our own convenience as we plan to build a higher-order deflation algorithm in Section 5 using the algorithm following the pattern established in this section.

One deflation step with fixed $\lambda$. The basic idea of the method is relatively simple. Let $\boldsymbol{\lambda} \in \mathbb{C}^{n}$ be a nonzero vector in $\operatorname{ker}\left(A\left(\boldsymbol{x}^{*}\right)\right)$, then the equations

$$
g_{i}(\boldsymbol{x})=\boldsymbol{\lambda} \cdot \nabla f_{i}(\boldsymbol{x})=\sum_{i=1}^{n} \lambda_{j} \frac{\partial f_{i}(\boldsymbol{x})}{\partial x_{j}}, \quad i=1,2, \ldots, N
$$

have $\boldsymbol{x}^{*}$ as a solution. Moreover, 
Theorem 5.1 The augmented system

$$
G(\boldsymbol{x})=\left(f_{1}, \ldots, f_{N}, g_{1}, \ldots, g_{N}\right)(\boldsymbol{x})=\mathbf{0}
$$

of equations in $\mathbb{C}[\boldsymbol{x}]$ is a deflation of the original system $F(\boldsymbol{x})=\mathbf{0}$ at $x^{*}$, i.e. $G\left(\boldsymbol{x}^{*}\right)=0$ and the multiplicity of the solution $x^{*}$ is lower in the new system.

The original proof of this statement in 14 uses the notion of a standard basis of the ideal $I=\left(f_{1}, f_{2}, \ldots, f_{N}\right)$ in the polynomial ring $R=\mathbb{C}[\boldsymbol{x}]$ w.r.t. a local order; this tool of computational commutative algebra can be used to obtain the multiplicity of $\boldsymbol{x}^{*}$, which is defined as the $\mathbb{C}$-dimension of the local quotient ring $R_{\boldsymbol{x}} / R_{x} I$.

On the other hand it is in correspondence with another way of looking at multiplicities - dual spaces of local functionals, so the proof can be written in that language as well (see Section 3).

One deflation step with indeterminate $\lambda$. Without loss of generality, we may assume corank $\left(A\left(\boldsymbol{x}^{*}\right)\right)=1$; consult [14 to see how the general case is reduced to this. Consider $N+1$ additional polynomials in $\mathbb{C}[\boldsymbol{x}, \boldsymbol{\lambda}]$ in $2 n$ variables:

$$
\begin{aligned}
g_{i}(\boldsymbol{x}, \boldsymbol{\lambda})=\boldsymbol{\lambda} \cdot \nabla f_{i}(\boldsymbol{x}) & =\sum_{j=1}^{n} \lambda_{j} \frac{\partial f_{i}(\boldsymbol{x})}{\partial x_{j}},(i=1,2, \ldots, N) \\
h(\boldsymbol{\lambda}) & =\sum_{j=1}^{n} b_{j} \lambda_{j}-1,
\end{aligned}
$$

where the coefficients $b_{j}$ are random complex numbers.

Theorem 5.2 Let $\boldsymbol{x}^{*} \in \mathbb{C}^{n}$ be an isolated solution of $F(\boldsymbol{x})=\mathbf{0}$ (in $\mathbb{C}[\boldsymbol{x}]$ ).

For a generic choice of coefficients $b_{j}, j=1,2, \ldots, n$, there exists a unique $\lambda^{*} \in \mathbb{C}^{n}$ such that the system

$$
G(\boldsymbol{x}, \boldsymbol{\lambda})=\left(f_{1}, \ldots, f_{N}, g_{1}, \ldots, g_{N}, h\right)(\boldsymbol{x}, \boldsymbol{\lambda})=0
$$

of equations in $\mathbb{C}[\boldsymbol{x}, \boldsymbol{\lambda}]$ has an isolated solution at $\left(\boldsymbol{x}^{*}, \boldsymbol{\lambda}^{*}\right)$.

The multiplicity of $\left(\boldsymbol{x}^{*}, \boldsymbol{\lambda}^{*}\right)$ in $G(\boldsymbol{x}, \boldsymbol{\lambda})=\mathbf{0}$ is lower than that of $\boldsymbol{x}^{*}$ in $F(\boldsymbol{x})=\mathbf{0}$.

Proof. Follows from Proposition 3.4 in [14.

Theorem 5.2 provides a recipe for the deflation algorithm: one simply needs to keep deflating until the solution of the augmented system corresponding to $\boldsymbol{x}^{*}$ becomes regular.

As a corollary we have that the number of deflations needed to make a singular isolated solution $\boldsymbol{x}^{*}$ regular is less than the multiplicity of $\boldsymbol{x}^{*}$. 


\subsection{Higher-Order Deflation with Fixed Multipliers}

We use the deflation operator to define an augmented system.

Theorem 5.3 Let $f_{1}, f_{2}, \ldots, f_{N}$ form a standard basis of $I$ w.r.t. the order opposite to $\succeq$. Consider the system $G^{(d)}(\boldsymbol{x})=\mathbf{0}$ in $\mathbb{C}[\boldsymbol{x}]$, where

$$
G^{(d)}(\boldsymbol{x})=\left\{\begin{array}{rc}
f_{j}(\boldsymbol{x}) & (j=1,2, \ldots, N) \\
g_{j, \alpha}(\boldsymbol{x}) & (j=1,2, \ldots, N,|\alpha|<d)
\end{array} .\right.
$$

(a) The system $G^{(d)}(\boldsymbol{x})=\mathbf{0}$ is a deflation of the original system $F(\boldsymbol{x})=\mathbf{0}$ at $\boldsymbol{x}^{*}$.

(b) Let $I=(F)$ and $J=\left(G^{(d)}\right)$ be the ideals generated by polynomials of the systems and $\succeq$ be a global monomial order on $\mathbb{Z}_{\geq 0}^{n}$. Then the following relation holds for initial supports

$$
\operatorname{in}_{\succeq}\left(D_{\mathbf{0}}[J]\right) \subset\left\{\beta-\beta_{Q} \mid \beta \in \operatorname{in}_{\succeq}\left(D_{\mathbf{0}}[I]\right)\right\} \cap \mathbb{Z}_{\geq 0}^{n},
$$

where $\beta_{Q}$ is the maximal element of the set $\operatorname{in}_{\succeq}\left(D_{\mathbf{0}}[I]\right) \cap\{\beta:|\beta| \leq d\}$.

Proof. Let $\boldsymbol{\lambda} \in \operatorname{ker}\left(A^{(d)}\left(\boldsymbol{x}^{*}\right)\right)$ be the vector used above to construct the operator $Q \in \mathbb{C}[\boldsymbol{\partial}]$ and the equations $g_{j, \alpha}(\boldsymbol{x})=0$.

First of all, $g_{j, \alpha}\left(\boldsymbol{x}^{*}\right)=\left.\left(Q \cdot\left(\boldsymbol{x}^{\alpha} f_{j}\right)\right)\right|_{\boldsymbol{x}=\boldsymbol{x}^{*}}=0$ provided $|\alpha|<d$ (by construction), hence, $\boldsymbol{x}^{*}$ is a solution to $G^{(d)}(\boldsymbol{x})=\mathbf{0}$.

To prove (a), it remains to show that the multiplicity drops, which follows from part (b) that is treated in the rest of this proof.

We shall assume for simplicity that $\boldsymbol{x}^{*}=\mathbf{0}$. This is done without the loss of generality using a linear change of coordinates: $\boldsymbol{x} \mapsto \boldsymbol{x}+\boldsymbol{x}^{*}$. It is important to note that in the new coordinates polynomials $Q \cdot\left(\boldsymbol{x}^{\alpha} f_{j}\left(\boldsymbol{x}+\boldsymbol{x}^{*}\right)\right)$ generate the same ideal as the polynomials $Q \cdot\left(\left(\boldsymbol{x}-\boldsymbol{x}^{*}\right)^{\alpha} f_{j}\left(\boldsymbol{x}+\boldsymbol{x}^{*}\right)\right)$.

Recall that $I=\langle F\rangle=\left\langle f_{1}, f_{2}, \ldots, f_{N}\right\rangle$, let $J=\left\langle G^{(d)}\right\rangle \supset I$ be the ideal generated by the polynomials in the augmented system. The reversed containment holds for the dual spaces: $D_{\mathbf{0}}[I] \supset D_{\mathbf{0}}[J]$.

There is a 1-to-1 correspondence between linear differential operators and linear differential functionals:

$$
\sum \lambda_{\beta} \partial^{\beta} \longleftrightarrow \sum \lambda_{\beta} \beta ! \Delta_{\beta}
$$

Let $\phi: \mathbb{C}[\boldsymbol{\partial}] \rightarrow D_{\mathbf{0}}$ and $\tau: D_{\mathbf{0}} \rightarrow \mathbb{C}[\boldsymbol{\partial}]$ be the corresponding bijections.

As in Section 3 we order terms $\Delta_{\beta}$ with $\succeq$, a global monomial order. Notice that since the choice of coefficients of the operator $Q$ is generic, $\beta_{Q}=\operatorname{in}_{\succeq}(Q)=$ $\operatorname{in}_{\succeq}(\phi(Q))$ is the maximal element of the set $\operatorname{in}_{\succeq}\left(D_{\mathbf{0}}[I]\right) \cap\{\beta:|\beta| \leq d\}$.

Next, we use the condition that $f_{i}$ form a standard basis. Since the corners of the staircase correspond to the initial terms of $f_{i}$, by Lemma 3.5 the staircase created with the corners at in ${ }_{\geq}\left(Q \cdot\left(\boldsymbol{x}^{\alpha} f_{i}\right)\right)$ bounds the set $\left\{\beta-\beta_{Q} \mid \beta \in\right.$ $\left.\operatorname{in}_{\succeq}\left(D_{\mathbf{0}}[I]\right)\right\} \cap \mathbb{Z}_{\geq 0}^{n}$, which, therefore, contains the initial support of $D_{\mathbf{0}}[J]$. 
Corollary 5.4 If there exist a local monomial order $\geq$ such that the minimal (standard) monomial in the set $\left\{\boldsymbol{x}^{\alpha} \notin \mathrm{in}_{\geq}(I):|\alpha| \leq d\right\}$ is minimal in the set of all standard monomials, then $\boldsymbol{x}^{*}$ is a regular solution of $G^{(d)}(\boldsymbol{x})=\mathbf{0}$.

Ideally we would like to be able to drop the assumption of the original polynomials forming a standard basis, since computing such a basis is a complex symbolic task, whereas our interest lies in the further numericalization of the approach. The following weaker statement works around this restriction.

Assuming $\boldsymbol{x}^{*}=\mathbf{0}$, let $\operatorname{supp}(F)=\bigcup_{j=1,2, \ldots, N} \operatorname{supp}\left(f_{j}\right)$.

Proposition 5.5 Assume $A(\mathbf{0})=\mathbf{0}$. Let $d_{0}=\min \left\{|\alpha|: x^{\alpha} \in \operatorname{supp}(F)\right\}$.

Then, in the notation of Theorem 5.3, for a generic deflating operator $Q$ the system $G^{(d)}(\boldsymbol{x})=\mathbf{0}$, where, $d<d_{0}$ is a deflation of the original system $F(\boldsymbol{x})=\mathbf{0}$ at the origin.

Moreover, if $d=d_{0}-1$ then the Jacobian of $G^{(d)}(\mathbf{0})$ is not equal to zero.

Proof. Fix a local monomial ordering that respects the degree. With the above assumptions, the initial ideal $\operatorname{in}(\langle F\rangle)$ will contain monomials of degree at least $d_{0}$. On the other hand, for a generic choice of the deflating operator $Q$ the $\operatorname{support} \operatorname{supp}\left(G^{(d)}\right)$ would contain a monomial of degree less than $d_{0}$. Therefore, there exists a monomial in $\operatorname{in}\left(\left\langle\operatorname{supp}\left(G^{(d)}\right)\right\rangle\right)$ that is not in $\operatorname{in}(\langle F\rangle)$, hence, $G^{(d)}$ is a deflation.

If $d=\left|d_{0}\right|-1$, then there is such monomial of degree 1 , which means that the Jacobian of the augmented system is nonzero.

Remark 5.6 Note that if the deflation order $d$ is as in Proposition 5.5, then it suffices to take an arbitrary homogeneous deflation operator of order $d$.

Next we explain the practical value of Proposition [5.5, Let $K=\operatorname{ker} A(\mathbf{0})$ and $c=\operatorname{corank} A(\mathbf{0})=\operatorname{dim} K$. Without a loss of generality we may assume that $K$ is the subspace of $\mathbb{C}^{n}$ has $\left\{x_{1}, \ldots, x_{c}\right\}$ as coordinates.

Now consider the system $F^{\prime}\left(x_{1}, \ldots, x_{c}\right)=F\left(x_{1}, \ldots, x_{c}, 0, \ldots, 0\right)$. This system has an isolated solution at the origin, and Proposition [5.5] is applicable, since the Jacobian is zero. Moreover, if we take the deflation of order $d=d_{0}-1$ of the original system $F$, with $d_{0}$ coming from the Proposition, the corank of the Jacobian the augmented system $G^{(d)}$ is guaranteed to be lower than that of $A(\mathbf{0})$.

Let us go back to the general setup: an arbitrary isolated solution $\boldsymbol{x}^{*}$, the Jacobian $A\left(\boldsymbol{x}^{*}\right)$ with a proper kernel $K$, etc. Algorithm 2.5 is a practical algorithm that can be executed numerically knowing only an approximation to $\boldsymbol{x}^{*}$. Proof. [Proof of correctness of Algorithm [2.5 for $\boldsymbol{x}^{0}=\boldsymbol{x}^{*}$.] We can get to the special setting of Proposition 5.5 in two steps. First, apply an affine transformation that takes $\boldsymbol{x}^{*}$ to the origin and ker $A\left(\boldsymbol{x}^{*}\right)$ to the subspace $K$ of $\mathbb{C}^{n}$ 
spanned by the first $c=\operatorname{corank} A\left(\boldsymbol{x}^{*}\right)$ standard basis vectors. Second, make a new system $F^{\prime}\left(x_{1}, \ldots, x_{c}\right)=0$ by substituting the $x_{i}=0$ in $F$ for $i>c$.

Let $\gamma^{\prime}=\left(\gamma_{1}^{\prime}, \ldots, \gamma_{c}^{\prime}\right) \in K$ be the image of the generic vector $\gamma$ under the linear part of the affine transform. Then $H(t)=F^{\prime}\left(\gamma_{1}^{\prime} t, \ldots, \gamma_{c}^{\prime} t\right)$.

Since $\gamma^{\prime}$ is generic, the lowest degree $d_{0}$ of the monomial in $\operatorname{supp}\left(F^{\prime}\right)$ is equal to $\min \left\{a \mid t^{a} \in \operatorname{supp} H(t)\right\}$. According to the Proposition 5.5 and the discussion that followed, $d=d_{0}-1$ is the minimal order of deflation that will reduce the rank of the system.

Remark 5.7 In view of Remark 5.6 it would be enough to use any homogeneous deflation operator of order $d$ :

$$
Q=\sum_{|\beta|=d} \lambda_{\beta} \partial^{\beta} \in \mathbb{C}[\boldsymbol{\partial}],
$$

such that the vector $\boldsymbol{\lambda}$ of its coefficients is in the kernel of the truncated deflation matrix, which contains only the rows corresponding to the original polynomials $F$ and only the columns labelled with $\partial^{\beta}$ with $|\beta|=d$.

\subsection{Indeterminate Multipliers}

As in Section 5.1, we now consider indeterminate $\lambda_{\beta}$. Now we should think of the differential operator $L(\boldsymbol{\lambda}) \in \mathbb{C}[\boldsymbol{\lambda}, \partial]$ and of additional equations $g_{j, \alpha}(\boldsymbol{x}, \boldsymbol{\lambda}) \in$ $\mathbb{C}[\boldsymbol{x}, \boldsymbol{\lambda}]$ as depending on $\boldsymbol{\lambda}$.

Proof of Theorem 2.4. Picking $m=\operatorname{corank}\left(A^{(d)}\left(\boldsymbol{x}^{*}\right)\right)$ generic linear equations $h_{k}$ guarantees that for $\boldsymbol{x}=\boldsymbol{x}^{*}$ the solution for $\boldsymbol{\lambda}$ exists and is unique; therefore, the first part of the statement is proved.

The argument for the drop in the multiplicity is similar to that of the proof of Theorem 5.2 .

\section{Computational Experiments}

We have implemented our new deflation methods in PHCpack [25] and Maple. Below we report on two examples.

One crucial decision in the deflation algorithm is the determination of the numerical rank, for which we may use SVD or QR in the rank-revealing algorithms. Both SVD and QR are numerically stable. We summarize the result from [4, page 118] for the problem of solving an overdetermined linear system $A \boldsymbol{x}=b$. The solution obtained by QR or SVD minimizes the residual $\|(A+\delta A) \widetilde{\boldsymbol{x}}-(b+\delta b)\|_{2}$ where the relative errors have the same magnitude as the machine precision $\epsilon$ :

$$
\max \left(\frac{\|\delta A\|_{2}}{\|A\|_{2}}, \frac{\|\delta b\|_{2}}{\|b\|_{2}}\right)=O(\epsilon)
$$




\subsection{Running Example 1}

To find initial approximations for the roots of the system (9), we must first make the system "square", i.e.: having as many equations as unknowns, so we may apply the homotopies available in PHCpack [25. Using the embedding technique of [20] (see also 21]), we add one slack variable $z$ to each equation of the system, multiplied by random complex constants $\gamma_{1}, \gamma_{2}$, and $\gamma_{3}$ :

$$
E(\boldsymbol{x}, z)=\left\{\begin{array}{c}
x_{1}^{3}+x_{1} x_{2}^{2}+\gamma_{1} z=0 \\
x_{1} x_{2}^{2}+x_{2}^{3}+\gamma_{2} z=0 \\
x_{1}^{2} x_{2}+x_{1} x_{2}^{2}+\gamma_{3} z=0 .
\end{array}\right.
$$

Observe that the solutions of the original system $F(\boldsymbol{x})=\mathbf{0}$ occur as solutions of the embedded system $E(\boldsymbol{x}, z)=\mathbf{0}$ with slack variable $z=0$. At the end points of the solution paths defined by a homotopy to solve $E(\boldsymbol{x}, z)=\mathbf{0}$, we find nine zeroes close to the origin. These nine approximate zeroes are the input to our deflation algorithm.

The application of our first deflation algorithm in [14 requires two stages. The Jacobian matrix of $F(\boldsymbol{x})=\mathbf{0}$ has rank zero at $(0,0)$. After the first deflation with one multiplier, the rank of the Jacobian matrix of the augmented system $G\left(\boldsymbol{x}, \boldsymbol{\lambda}_{1}\right)=\mathbf{0}$ equals one, so the second deflation step uses two multipliers. After the second deflation step, the Jacobian matrix has full rank, and $(0,0)$ has then become a regular solution. Newton's method on the final system then converges again quadratically and the solution can be approximated efficiently with great accuracy. Once the precise location of a multiple root is known, we are interested in its multiplicity. The algorithm of [3] reveals that the multiplicity of the isolated root equals seven.

Starting at a root of low accuracy, at a distance of $10^{-5}$ from the exact root, the numerical implementation of Algorithm 2.5 predicts two as the order, using $10^{-4}$ as the tolerance for the vanishing of the coefficients in the univariate interpolating polynomial. The Jacobian matrix of the augmented system $G^{(2)}$ has full rank so that a couple of iterations suffice to compute the root very accurately.

\subsection{A Larger Example}

The following system is copied from [12]:

$$
F(\boldsymbol{x})=\left\{\begin{array}{c}
2 x_{1}+2 x_{1}^{2}+2 x_{2}+2 x_{2}^{2}+x_{3}^{2}-1=0 \\
\left(x_{1}+x_{2}-x_{3}-1\right)^{3}-x_{1}^{3}=0 \\
\left(2 x_{1}^{3}+2 x_{2}^{2}+10 x_{3}+5 x_{3}^{2}+5\right)^{3}-1000 x_{1}^{5}=0 .
\end{array}\right.
$$

Counted with multiplicities, the system has 54 isolated solutions. We focus on the solution $(0,0,-1)$ which occurs with multiplicity 18 .

Although Algorithm 1 suggests that the first-order deflation would already lower the corank of the system, we would like to search for a homogeneous deflation operator $Q$ of order two. 
To this end we construct the (truncated) deflation matrix $\bar{A}\left(x_{1}, x_{2}, x_{3}\right)$ with has 12 rows and only 6 columns, which correspond to $\left\{\partial_{1}^{2}, \partial_{1} \partial_{2}, \partial_{1} \partial_{3}, \partial_{2}^{2}, \partial_{2} \partial_{3}, \partial_{3}^{2}\right\}$.

The kernel of $\bar{A}(0,0,-1)$ is spanned by $(1,6,8,-3,0,4)^{T}$ and $(0,3,3,-1,1,2)^{T}$. The operator corresponding to the former,

$$
Q=\partial_{1}^{2}+6 \partial_{1} \partial_{2}+8 \partial_{1} \partial_{3}-3 \partial_{2}^{2}+4 \partial_{3}^{2},
$$

regularizes the system, since the equations

$$
\left\{\begin{array}{l}
Q \cdot\left(x_{1} f_{1}\right)=8 x_{1}+24 x_{2}+16 x_{3}+16=0 \\
Q \cdot\left(x_{2} f_{1}\right)=24 x_{1}-24 x_{2}+16 x_{3}+16=0 \\
Q \cdot\left(x_{3} f_{1}\right)=32 x_{1}=0
\end{array}\right.
$$

augmented to the original equations, give a system with the full-rank Jacobian matrix at $(0,0,-1)$.

\section{Conclusion}

In this paper we have described two methods of computing the multiplicity structure at isolated solutions of polynomial systems. We have developed a higher-order deflation algorithm that reduces the multiplicity faster than the first-order deflation in [14].

In our opinion, one of the main benefits of the higher order deflation for the numerical algebraic geometry algorithms is the possibility to regularize the system in a single step. For that one has to determine the minimal order of such a deflation or, even better, construct a sparse ansatz for its deflation operator. Predicting these numerically could be a very challenging task, which should be explored in the future.

\section{References}

[1] E.L Allgower, K. Böhmer, A. Hoy, and V. Janovský. Direct methods for solving singular nonlinear equations. ZAMM Z. Angew. Math. Meth., 79(4):219-231, 1999.

[2] C. Bates, D.J. Peterson and A.J. Sommese. A numerical-symbolic algorithm for computing the multiplicity of a component of an algebraic set. $J$. Complexity, 22(4):475-489, 2006.

[3] B.H. Dayton and Z. Zeng. Computing the multiplicity structure in solving polynomial systems. In M. Kauers, editor, Proceedings of the 2005 International Symposium on Symbolic and Algebraic Computation, pages 116-123. ACM, 2005.

[4] J.W. Demmel. Applied Numerical Linear Algebra, volume 45 of Classics in Applied Mathematics. SIAM, 2003. 
[5] P. Deuflhard. Newton Methods for Nonlinear Problems. Affine Invariance and Adaptive Algorithms. Springer-Verlag, 2004.

[6] W.J.F. Govaerts. Numerical Methods for Bifurcations of Dynamical Equilibria. SIAM, 2000.

[7] D. Grayson and M. Stillman. Macaulay 2, a software system for research in algebraic geometry. Available at http://www.math.uiuc.edu/Macaulay2/.

[8] G.-M. Greuel and G. Pfister. Advances and improvements in the theory of standard bases and syzygies. Arch. Math., 66:163-196, 1996.

[9] G.-M. Greuel and G. Pfister. A Singular Introduction to Commutative Algebra. Springer-Verlag, 2002.

[10] G.-M. Greuel, G. Pfister, and H. Schönemann. Singular 2.0. A Computer Algebra System for Polynomial Computations, Centre for Computer Algebra, University of Kaiserslautern, 2001. http://www.singular.uni-kl.de.

[11] P. Kunkel. A tree-based analysis of a family of augmented systems for the computation of singular points. IMA J. Numer. Anal., 16:501-527, 1996.

[12] G. Lecerf. Quadratic Newton iteration for systems with multiplicity. Found. Comput. Math., 2:247-293, 2002.

[13] A. Leykin, J. Verschelde, and A. Zhao. Evaluation of Jacobian matrices for Newton's method with deflation to approximate isolated singular solutions of polynomial systems. To appear in D. Wang and L. Zhi, editors, SNC 2005 Proceedings. International Workshop on Symbolic-Numeric Computation. Xi'an, China, July 19-21, 2005.

[14] A. Leykin, J. Verschelde, and A. Zhao. Newton's method with deflation for isolated singularities of polynomial systems. Theoretical Computer Science, 359(1-3):111-122, 2006.

[15] Y. Lijun. On the generalized Lyapunov-Schmidt reduction. ZAMM Z. Angew. Math. Mech., 84(8):528-537, 2004.

[16] F.S. Macaulay. The Algebraic Theory of Modular Systems. Cambridge University Press, 1916. Reissued with an Introduction by Paul Roberts in the Cambridge Mathematical Library 1994.

[17] F. Mora. An algorithm to compute the equations of tangent cones. In J. Calmet, editor, Computer Algebra. EUROCAM'82, European Computer Algebra Conference. Marseille, France, April 1982., volume 144 of Lecture Notes in Computer Science, pages 158-165. Springer-Verlag, 1982.

[18] B. Mourrain. Isolated points, duality and residues. Journal of Pure and Applied Algebra, 117/118:469-493, 1997. 
[19] T. Ojika, S. Watanabe, and T. Mitsui. Deflation algorithm for the multiple roots of a system of nonlinear equations. J. Math. Anal. Appl., 96:463-479, 1983.

[20] A.J. Sommese and J. Verschelde. Numerical homotopies to compute generic points on positive dimensional algebraic sets. J. of Complexity, 16(3):572602, 2000.

[21] A.J. Sommese and C.W. Wampler. The Numerical solution of systems of polynomials arising in engineering and science. World Scientific, 2005.

[22] H.J. Stetter. Numerical Polynomial Algebra. SIAM, 2004.

[23] H.J. Stetter and G.T. Thallinger. Singular systems of polynomials. In O. Gloor, editor, Proceedings of the 1998 International Symposium on Symbolic and Algebraic Computation, pages 9-16. ACM, 1998.

[24] G.T. Thallinger. Zero Behavior in Perturbed Systems of Polynomial Equations. PhD thesis, Tech. Univ. Vienna, 1998.

[25] J. Verschelde. Algorithm 795: PHCpack: A general-purpose solver for polynomial systems by homotopy continuation. ACM Trans. Math. Softw., 25(2):251-276, 1999. Software available at http://www.math.uic.edu/ ${ }^{j}$ an.

[26] J. Verschelde and A. Zhao. Newton's method with deflation for isolated singularities. Poster presented at ISSAC'04, 6 July 2004, Santander, Spain. Available at http://www.math.uic.edu/ jan/poster.pdf and at http://www.math.uic.edu/ azhao1/poster.pdf. 